\title{
Considerations in developing and delivering a non-pharmacological intervention for symptom management in lung cancer: the views of health care professionals
}

\author{
Richard Wagland • Jackie Ellis • Chris D. Bailey • \\ Jemma Haines • Ann Caress • Mari Lloyd Williams • \\ Paul Lorigan - Jaclyn Smith • Carol Tishelman • \\ Richard Booton • Karen Luker • Fiona Blackhall • \\ Alex Molassiotis
}

Received: 7 July 2011 / Accepted: 26 December 2011 / Published online: 20 January 2012

(C) Springer-Verlag 2012

\begin{abstract}
Background A respiratory distress symptom cluster has recently been identified in lung cancer associated with breathlessness, cough and fatigue, and the study reported here is part
\end{abstract}

R. Wagland $\cdot$ C. D. Bailey

Faculty of Health Sciences, University of Southampton,

Highfield Southampton SO17 1BJ, UK

\section{A. Caress $\cdot$ K. Luker $\cdot$ A. Molassiotis $(\bowtie)$}

School of Nursing, Midwifery and Social Work,

University of Manchester,

Manchester M13 9PL, UK

e-mail: alex.molassiotis@manchester.ac.uk

J. Smith $\cdot$ R. Booton

Respiratory Research Group, School of Translational Medicine, University of Manchester,

Manchester M13 9PL, UK

J. Ellis • M. L. Williams

Academic Palliative and Supportive Care Studies Group (APSCSG), Division of Primary Care, University of Liverpool, Liverpool, UK

\section{J. Haines}

Medical Directorate, Lancashire Teaching Hospitals,

Preston, Lancashire, UK

P. Lorigan $\cdot$ F. Blackhall

Christie NHS Foundation Trust,

Manchester, UK

C. Tishelman

Department of Learning, Informatics, Management and Ethics, Karolinska Institutet,

Solna, Sweden of a wider body of work being undertaken to develop a novel non-pharmacological intervention (NPI) for the management of this symptom cluster. The current paper reports the views of health care professionals (HCPs) involved with cancer care regarding the most appropriate ways of developing and delivering such a novel intervention.

Methods Five focus groups, supplemented with additional telephone interviews, were conducted with a range of both community- and acute-based HCPs involved in symptom management for lung cancer patients. Participants included oncologists, palliative care consultants, specialist nurses, occupational therapists and physiotherapists. The focus groups were transcribed verbatim and analysed using NVIVO to support a framework analysis approach.

Results The current delivery of NPIs was found to be ad hoc and varied between sites both in terms of what was delivered and by which health care professionals. The provision of NPIs within acute medical settings faced common problems concerning staffing time and space, and there was a recognition that the preference of most patients to make as few hospital visits as possible also complicated NPI teaching. Moreover, there may only be a small window of opportunity in which to effectively teach lung cancer patients a novel NPI as the period between diagnosis and the onset of severe symptoms is often short.

Discussion The participants agreed that the novel symptom management NPI should be individually personalised to the needs of each patient and be available for patients when they become receptive to it. Moreover, they agreed that the intervention would be most effective if delivered to patients individually rather than in groups, outside acute medical 
settings where possible and closer to patient's homes, should be delivered by an HCP rather than a trained volunteer or lay person and should involve informal carers wherever practicable.

Keywords Non-pharmacological $\cdot$ Lung cancer $\cdot$ Symptom management $\cdot$ Symptom cluster

\section{Introduction}

Symptom management is a priority for cancer patients and is an important component of improving quality of life for lung cancer patients $[13,26]$; yet, it remains one of the most striking areas of unmet needs [42]. Symptom distress is a strong predictor of survival [12], and lung cancer patients are found to experience more symptom distress than patients with other types of cancer [7], with cough and breathing changes being the most common symptoms experienced [9]. Patients with cancer rarely present with a single symptom and more often than not experience several concurrently. Recognition of this has recently led to an increasing research focus on 'symptom clusters' rather than single symptoms $[15,23,32,33]$. Understanding such clusters may improve symptom management, and several studies have identified the presence of a salient cluster of symptoms experienced by patients with lung cancer [5, 20,21, 35], showing close associations among breathlessness, cough and fatigue.

Respiratory symptoms are often inadequately relieved by pharmacological interventions, which may also cause adverse effects [3, 7]. Bailey et al. [2] have argued that there is a need for more investigation of promising non-pharmacological interventions (NPIs), and a recent Cochrane review on wellbeing and quality of life interventions in lung cancer identified only one non-pharmacological programme in managing breathlessness effectively [3]. This 'Corner model' $[4,10]$ is a 3-6 weeks programme involving counselling, breathing retraining, relaxation and teaching coping and adaptation strategies delivered by a nurse practitioner. Despite criticism directed at the programme's reliance on specialist nurses, high costs and an exclusive focus on a single symptom [25], the Corner programme has nevertheless shaped nurse-led, nonpharmacological breathlessness care for lung cancer in the UK [40]. Other NPIs that have shown promise in respiratory diseases (particularly asthma and obstructive pulmonary diseases) include education, psychological interventions and support, individualised care plans, inspiratory muscle training and physical activity [2]. Such approaches together with breathing exercises are commonly included in pulmonary rehabilitation and they may be transferable and effective in the lung cancer settings. This assertion, however, will require further testing. In contrast, while fatigue is often managed by NPIs [11, 28], no NPIs have been tested with cough in lung cancer patients, and a recent Cochrane systematic review of cough management found no documented NPIs in lung cancer [34], a symptom which is often distressing and impacts on quality of life [36]. Thus, there is a need to extend the remit of symptom management intervention research to encompass symptoms thus far neglected as part of symptom clusters and in particular the breathlessness-cough-fatigue cluster which often leads to distress for patients with lung cancer.

\section{Background to wider study}

This paper derives from an ongoing body of work aiming to develop a novel NPI for the management of breathlessnesscough-fatigue cluster identified in lung cancer. An NPI specifically designed to address this symptom cluster may include such techniques as: education and advice, breathing exercises, vocal hygiene, cough suppression, sleep hygiene, exercise and energy conservation and anxiety management. As such, it is one of the first studies focusing on the management of a symptom cluster rather than single symptoms. However, significant challenges have been experienced in previous trials with lung cancer patients due to their often low performance status and high symptom burden with complex symptomatology, which can lead to difficulties in recruitment and retention as well as in implementing NPIs in practice $[27,46]$. The impetus for developing a new intervention stems from the fact that patients/caregivers have not been thoroughly consulted in the past about their preferences and that past interventions are complex and intensive, raising concerns about their implementation in routine clinical practice [46]. We therefore deemed it imperative to first explore the views of patients, informal caregivers and health professionals about issues that could make an intervention more appropriate and viable and thus stand a better chance of being integrated into practice prior to testing any intervention. Data from the patients and caregivers suggested that ten elements of an intervention are critical considerations, including the perceived relevance of potential techniques, appreciable benefits in the short term, convenience (being part of daily routine), flexibility to accommodate variation in patient preferences of intervention components, beliefs about the intervention, timing of the intervention, venue, caregiver involvement, the provider of the intervention (being a health professional) and training format/contact with other patients [17]. This paper reports on the data from the health care professionals.

\section{Methods}

The study and management of cancer-related symptoms benefits from a multi-disciplinary approach, including views of a broad range of health care professionals (HCPs) in modelling a complex symptom management intervention [16]. Focus 
group discussions (FGDs) were used for data collection as they explore collective rather than individual experiences and reveal the nature and variety of participants' views [29]. Indeed FGDs arguably may yield more and richer information than individual interviews with the same participants [31]. A purposive sampling approach facilitated access to the views of the necessarily wide variety of key informants involved in the care and treatment of patients with lung cancer [31], including palliative care consultants and oncologists, physiotherapists, occupational therapists and specialist nurses.

Research associates contacted lead consultants, introduced the study and secured assistance with the identification of a range of HCPs involved with palliative and cancer care. Individuals were then approached individually to determine willingness to be involved and availability. We aimed to conduct FGDs with participants of mixed professional backgrounds to allow contrasting opinions to emerge between the represented professions [31]. However, it proved difficult to recruit physiotherapists to the first four focus groups, so a further group interview was arranged in one hospital wth physiotherapists $(n=4)$. The focus groups were supplemented with telephone interviews with HCPs from different backgrounds to further validate findings [45]. These interviews were conducted with an oncologist, a clinical nurse specialist, a physiotherapist and an occupational therapist. Most interviews with FGDs (FGDs 1, 3, 4, 5) lasted between 1 and $1.5 \mathrm{~h}$, although that with one FGD took $2 \mathrm{~h}$ and $15 \mathrm{~min}$.

Four experienced qualitative researchers were involved with running the focus groups, with one researcher acting as the moderator in each of the three sites and being supported in each area by another local researcher who acted as observer and notetaker. Initially, we developed a FGD topic guide to address issues relating to the delivery, uptake, future implementation and cost-effectiveness of an NPI including the perception of the optimum point within treatment and disease progression for delivery of the NPI, whether the intervention should be delivered by a trained volunteer or a HCP, whether delivery should be provided within a hospital or nearer home, whether the intervention should be delivered to patients individually or in groups and the degree to which informal caregivers should be involved with the delivery process. The topic guide also included probes of the perceptions of HCPs with regard to the impact of symptom clusters on patients and current or possible management strategies.

The FGDs were transcribed verbatim and analysed using the computer programme NVivo for assistance in structuring a framework analysis [43]. Framework analysis aims at facilitating applied research and its requirements to meet specific information needs and actionable outcomes and is conducted through a series of stages. The FGD moderator and interviewer led the analysis, ensuring deep familiarisation with the data. To facilitate data analysis, each of the four researchers independently analysed one of the focus groups that took place within their area and discussed their findings with the full research team to agree upon an index of emerging themes. Thereafter, regular weekly discussions took place between the four researchers involved with the focus groups to review the development of the thematic framework and ensure analytical rigour. A thematic framework was developed, initially drawn from the topic guide, to identify the key concepts central to the design of a novel NPI to help patients with lung cancer and their carers manage the breathlessness-cough-fatigue symptom cluster. During the analysis, other categories were derived from the data including views concerning the level of skill and knowledge necessary to deliver NPIs; factors impacting upon NPI effectiveness such as consistency of approach; the impact of resource constraints such as time, space and skilled staff; the characteristics of the disease and assumptions concerning the characteristics of patients with lung cancer. The whole data set was then indexed according to these categories (indexing) and comparisons were made both within and between them according to their thematic content (charting). Relationships and associations between the categories were then identified (mapping and interpretation) that explained why HCPs favoured certain features of a novel NPI over others. For instance, it became clear that the views of the participants concerning the skills required, the factors associated with effectiveness and the complex symptomatology of the disease all had strong implications for the mode of delivery that the intervention should take.

The study was approved by a multi-site Research Ethics Committee (REC Ref: 09/H1011/31) and all participating hospitals.

\section{Findings}

In total, $43 \mathrm{HCPs}$ working in community, acute specialist and palliative care in six NHS trusts in the northwest and south of England were approached and invited to participate in the study. Thirty were successfully recruited to participate in five focus groups, with an additional four HCPs individually interviewed. Table 1 indicates the size and composition of each of the focus groups, which included specialist nurses $(n=$ $19)$, physiotherapists $(n=5)$, occupational therapists $(n=2)$, oncologists $(n=4)$ and palliative care consultants $(n=4)$.

\section{Current standard care}

Participants from the six trusts reported that for most lung cancer patients there was no systematic approach to nonpharmacological symptom management. Specialised breathlessness clinics were available at only half of the trust hospitals. Even where clinics did exist, oncologists often appeared unaware of them and agreed that they were probably under-used. 
Table 1 Composition of focus groups

\begin{tabular}{|c|c|c|c|}
\hline Focus group & Invited & Recruited & Constitution \\
\hline $\begin{array}{l}\text { Focus group } \\
1 \text { (from two } \\
\text { hospitals) }\end{array}$ & 11 & 7 & $\begin{array}{l}\text { Hospital palliative care team } \\
\text { CNS x } 3 \\
\text { Community palliative care } \\
\text { team CNSs } x 2 \\
\text { Palliative care consultant } x 1 \\
\text { Palliative care registrar } \mathrm{x} 1\end{array}$ \\
\hline $\begin{array}{l}\text { Focus group } \\
2 \text { (from two } \\
\text { hospitals) }\end{array}$ & 9 & 6 & $\begin{array}{l}\text { Lung cancer CNS x4 } \\
\text { Occupational therapist } \mathrm{x} 1 \\
\text { Medical oncologist } \mathrm{x} 1\end{array}$ \\
\hline $\begin{array}{l}\text { Focus group } \\
3 \text { (from one } \\
\text { hospital) }\end{array}$ & 11 & 7 & $\begin{array}{l}\text { Nurse consultant } \mathrm{x} 1 \\
\text { Palliative care consultants } \mathrm{x} 2 \\
\text { Clinical oncologist } \mathrm{x} 1 \\
\text { Macmillan lung CNSs x2 }\end{array}$ \\
\hline $\begin{array}{l}\text { Focus group } \\
4 \text { (from one } \\
\text { hospital) }\end{array}$ & 8 & 6 & $\begin{array}{l}\text { Research lead nurse x1 } \\
\text { HPCT CNS x } 5 \\
\text { Medical oncologist x } 1\end{array}$ \\
\hline $\begin{array}{l}\text { Focus group } \\
5 \text { (from one } \\
\text { hospital) }\end{array}$ & 4 & 4 & $\begin{array}{l}\text { CPCT physiotherapists } x 3 \\
\text { HPCT physiotherapist } x 1\end{array}$ \\
\hline $\begin{array}{l}\text { Individual } \\
\text { interviews }\end{array}$ & & 4 & $\begin{array}{l}\text { Medical oncologist } \mathrm{x} 1 \\
\text { Clinical nurse specialist } \mathrm{x} 1 \\
\text { Physiotherapist } \mathrm{x} 1 \\
\text { Occupational therapist } \mathrm{x} 1\end{array}$ \\
\hline Total & 43 & $30(+4)=34$ & \\
\hline
\end{tabular}

HPCT hospital palliative care team, $C P C T$ community palliative care team

The participants were rarely aware of any specialised clinics for cough or fatigue. Indeed it was reported that it was normally only patients with serious symptoms, for whom pharmacological treatment was having little effect, that were referred for non-pharmacological symptom management, and this would usually be delivered by physiotherapists or specialist nurses within hospital or community palliative care teams. Highly symptomatic patients could sometimes also be referred to symptom management clinics near their homes, often within hospices and day care services. Overall, HCPs agreed that breathlessness-cough-fatigue are currently inadequately addressed in standard care as illustrated by the following exchange between consultants in one FG:

Palliative care consultant: 'I think a lot of our work tends to be in response to sick inpatients, where a clinical situation may be changing, and yeah, dealing with the anxiety becomes an important component. But perhaps we don't tap into formal breath control training as much as there is potential for.'

Oncologist: 'I think it's even more underused, and we probably don't...certainly unless somebody has a major problem, and they're identified as having very difficult control issues or whatever, I may not question this enough, and I wasn't aware of some of the routes that I could've gone down. I usually would refer to the palliative care team and refer to my respiratory colleagues and the hospice. And I wasn't aware that there was a separate breathlessness clinic that I could've gone down. ...It might be that we can improve a lot more people, they could get some benefit from [NPIs] that perhaps people like myself haven't identified. I mean it's only really the very difficult cases that often get sent on.'-(FGD 3)

\section{Positive attitudes towards NPIs amongst HCPs}

There was broad support amongst all participants that NPIs can provide important psychological support for patients, providing them with the means to assert some control over their symptoms, especially those related to anxiety. This is illustrated in the following discussion between specialist nurses, although there is also a note of caution that for NPIs to be effective patients need to be receptive to them:

Specialist nurse 1: I think it comes down to personalities, and you're talking the patient through the [nonpharmacological] techniques and advise them, and watch them do the breathing techniques, but whether they actually [use them].... And the people who have gone away and done it have said to me that it has helped them. I don't know what you've found?

Specialist nurse 2: I think so that patients feel empowered, they've got this little toolbox so their anxiety is actually lessened because they think 'I know how to deal with this', and so...

Specialist nurse 1: Because they feel in control

Specialist nurse 2: Exactly.

(FGD 2)

There was also consensus that NPIs should play an integral role in the management of symptoms alongside pharmacological interventions and that interventions should be presented to patients and their carers as a central element within their treatment. Nevertheless, some caution was expressed by some HCPs that although there was undoubted potential benefit, patients should not be led to have unrealistic expectations of what NPIs could achieve as a palliative medicine consultant explains:

'I mean there's definitely a role [for NPIs], and we've heard of a range of techniques from complementary therapies to breathlessness, relaxation techniques, et cetera. I think it's obviously something that has to be most likely [provided] alongside everything else, and the patients often will need some form of medication 
to help control the symptoms. But no, it's a valuable part of treatment in palliative care.'

(FGD 4)

\section{Non-pharmacological interventions should be personalised and delivered by health care professionals}

The participants indicated a number of reasons why caution was necessary when using NPIs, arguing that the facilitation of patients to learn them should be undertaken by HCPs rather than volunteers or lay persons. Participants reported that the diaphragmatic breathing technique, for example, may sometimes be inappropriate for particularly anxious patients if they found it difficult to perform because it may lead to frustration which could exacerbate breathing difficulty. Patients may also have co-morbidities such as emphysema that might make diaphragmatic breathing inappropriate. Similarly, several participants believed that exercise programmes can sometimes over-burden or pressurise patients. Fatigue is not physically obvious and can often be under-estimated by the lay person, possibly resulting in the patient being asked to do more than is optimal. Some participants also argued that a fine line existed between exercise being beneficial for fatigue and harmful for breathlessness. Furthermore, the participants said that teaching anxiety management was particularly demanding because it required a close relationship of trust with the patient and that discussing patient anxieties often uncovered a number of latent fears that required good counselling skills to be adequately addressed.

Most participants agreed, therefore, that it was necessary for the individuals delivering the intervention to be able to understand the underlying pathology of a symptom (whether it is caused by the disease or treatment), to determine in each case whether particular strategies were likely to be effective and to provide ongoing assessment.

'I think it takes a level of skill to understand what the patient's problem is in front of you, and what they are able to do with you. ... So you can see how a patient is responding to your instructions, and then you can change what you are asking them to do while you are with them.'

Telephone interview 4 (physiotherapist)

Respiratory distress symptoms are interrelated and it was thought that 'simplistic and generic' advice from nonprofessionals would not lead to effective management of those symptoms. Rather, most participants believed that any novel NPI developed for the management of respiratory distress symptoms should be personalised for each individual patient, especially as respiratory symptoms may be dynamic and changing over time.
Palliative medicine consultant: 'And I hope that your package [of NPI strategies] would allow a degree of individual tailoring...'

Specialist nurse: 'Because we could spend the next hour all of us telling you anecdotes about the patient who found that this is the best thing for them or this is, all on non-drug strategies, and that it's different things for different people, and it's being able to have a menu, for want of a better word, of things that we can talk to, and teach the person who is ill and their family, that we're comfortable with, ...that then they can feel in control of, 'cause [we're] saying some people find this helps, some people find this helpful, other people find this helps, or you might find this helps, which of those do you think you want to try? And then they're in control already.'

Palliative medicine consultant: '...it's all about tailoring the package.'

(FGD 1)

The participants overwhelmingly suggested that physiotherapists would be the professional group most effective at facilitating patients to learn NPIs. In-patients and community palliative care patients are often referred to physiotherapists if they have mild to serious respiratory symptoms, and the participants said that physiotherapists generally had the best training to impact beneficially upon patients, as one oncology consultant said:

'I think with physios it's amazing some of the things that they can do, and I think I definitely underutilise them, because they're not in many of my clinics, any of my clinics. And I certainly found that some people who became an inpatient here, maybe [for] a slightly different reason had been picked up by physios, and then afterwards they made quite a lot of improvement [in symptoms] because of that.'

(FGD 4)

However, physiotherapists are rarely present in out-patient clinics, and while occupational therapists were also very effective in delivering NPIs, they were usually only involved with discharge planning for in-patients. Oncologists did not perceive the teaching of NPIs as part of their remit, and it was more broadly accepted amongst participants that patients themselves had a different relationship with and expectations of oncologists than other allied medical staff. Palliative medicine consultants generally accepted facilitating patients to learn NPIs to be part of their role but were nevertheless concerned that they would not have sufficient time during consultations to effectively deliver the proposed intervention. In contrast, specialist and palliative care nurses were thought to have more interaction with patients than had other HCPs and were thus better placed to deliver an NPI. The participants 
concluded, as illustrated separately by both a specialist nurse and an oncologist below, that the delivery of an NPI would most likely, by default, be undertaken by specialist nurses.

'I mean the patients we see, often when they come into clinic, it's a very medical lead time really, and it's a very specific time. ... So I would have thought nurses would probably have more interaction with patients to be able to do those things and talk about those things really.'

Telephone interview 1: clinical specialist nurse (CNS)

'I think sometimes [patients] see, certainly like the oncologists, they see as [having] a different role, and they maybe can't tell us some things. And I think maybe that educating the lung cancer specialist nurses to be able to pick up some of these [NPIs]. I don't think [doctors] are going to see everybody, and so [CNSs] are the people who would see everyone.'

(FGD 3) Oncology consultant

\section{Factors influencing the effectiveness of non-pharmacological interventions}

Several participants argued that the effectiveness of an intervention depends not just upon the competence of the facilitator but also upon both the patient and facilitator believing in its potential effectiveness. The participants said that patients are more likely to believe in the effectiveness of an intervention if delivered by an HCP and if it is tailored to their individual needs. However, for the patient's confidence in effectiveness to be maintained, each HCP delivering an NPI should adhere to an approach consistent with that used by their colleagues and be confident in their own ability to deliver it. Many participants said that these fundamental conditions for effectiveness were often currently absent. Indeed several participants indicated that without additional training they lacked the confidence to lead NPIs directed at the breathlessness-coughfatigue cluster. This is exemplified in this exchange between two specialist nurses:

Specialist nurse 1: 'I think what would be really useful, to have training to know how to target particular symptoms, and what advice to give [patients] because we see people during the treatment phase of the illness and then follow up....'

Specialist nurse 2: 'I think for me, I mean this might just be because I'm so new in post, but if someone specifically came to me and had specific concerns around their breathing or coughing, I probably wouldn't be able to list everything and say 'have you tried this?' I think maybe an informal session on diaphragmatic breathing, for me, that would be something that I would need training around.'

(FGD 4)

\section{Optimum time of intervention delivery}

The optimum point during a patient's treatment and disease progression for delivery of an NPI related to a participant's considerations about both the disease itself and the perceived characteristics of patients with lung cancer in general. Most participants said that patients needed to be receptive to the potential benefits of the NPI, which was usually only possible once they were symptomatic. However, this was said to be paradoxical since the participants also agreed that the more severe a patient's symptoms became the more difficult it would be for the intervention to be effective. Thus, there may only exists a small window of opportunity for patients to learn the various techniques:

'I think the problem is we often get people referred to us very late in their disease, and there isn't time to use those strategies really. People want a quick fix because they're very symptomatic and sometimes we have no choice really to do anything but pharmacological interventions.'

Telephone interview 1: CNS

'You have a very small window between them being okay and thinking they don't need [NPIs]... and then being really ill and being terminal, about to die.'

(FGD 1) Palliative care consultant

The participants were unanimous in agreeing that it would be inappropriate to discuss NPIs with patients at the point of diagnosis as there was already much new information patients received regarding their disease, prognosis and treatment and because time is so limited in acute health care settings. Rather, the participants suggested that the intervention should generally be introduced once the patients completed their first cycle of treatment, provided that symptom onset does not make earlier use of the NPI more appropriate.

'So you can imagine, you've already explained the side effects of chemotherapy which they will not retain at all, we give written information for this purpose. If you start telling them about breathing techniques, relaxation techniques, they will never remember [them].'

(FGD 4) Oncology consultant

The participants also noted that many patients with lung cancer were often reluctant to discuss their symptoms; they interpreted this being either because symptoms were believed to be a normal part of the disease or an indication of disease progression. It was also explained that the patients may be frightened to admit to worsening symptoms because they fear 
the staff might prematurely decide treatment was ineffective and hence terminate it. Moreover, many participants agreed that patients tended to focus upon pharmacological treatments, especially in the early stages of their disease. Another common discussion amongst FGD participants was that patients with lung cancer were generally more dismissive of the potential effect of non-pharmacological interventions than perhaps with other cancer groups. This tendency was speculatively ascribed to socio-economic and demographic factors, with comparisons often made with breast cancer patients who were perceived to be more receptive to non-pharmacological approaches.

'I offer [NPIs] far more than it's taken up. I mean most patients go, 'Well, that's not really for me.' ... And a lot of these patients, they just aren't in for those [NPI] therapies as much with lung cancer as maybe other types of cancer. But I think it's a social thing as well because a lot of the patients in this disease group come from more lower socio-economic classes, and they don't maybe access those kinds of support normally, so it's new for them.'

\section{(FGD 4) CNS}

The participants overwhelmingly said the intervention should be delivered when the patient was symptomatic but also psychologically ready to confront their symptoms and able to be receptive to the technique. This means that not only should the strategies and techniques that constitute the intervention be personalised to each patient but also that the point at which the intervention was delivered would depend upon each individual. A specialist nurse explains:

'...this has got to be done in, you know, the second appointment after the diagnosis or this has got to be done at the first appointment post-treatment. Because you're going to get some patients who present really short of breath and whatever you do is not going to make a difference, and you get other patients who don't get short of breath and fatigued until right when they're terminally ill and about to die.'

(FGD 1)

Furthermore, as many patients may not retain all the information they initially receive regarding the intervention, most participants believed that the teaching of NPIs should be repeated at least once. Again, as the following quote from a specialist nurse indicates, some skill would be necessary to assess how much of the intervention the patient had understood and how effective the techniques had been:

'I think it will vary from patient to patient, and you've got some that will pick it up really, really easily, and others that will just take longer, and some people that may not get it at all. You certainly need a couple of sessions with everybody to give them information and then they'll go away with it, try it and come back and you can assess them. But some people might need another follow-up if they weren't too good on that.' (FGD 4) CNS

\section{Delivering non-pharmacological interventions within the community}

The participants agreed that the location for the delivery of the intervention should be an environment in which the patient is optimally relaxed, and most suggested this would more likely be a patient's home rather than within a medical facility. Many participants said that patients waiting for consultant appointments are often too anxious to be able to learn NPIs, and several participants also noted that patients often lack affordable transport to make repeat visits to outpatient clinics. Logistical problems were also indicated by participants that make it difficult for staff to deliver NPIs in a hospital setting, such as securing an appropriate room and scheduling protected nursing time.

'I suppose where it happens is where the patient is most comfortable really isn't it? Maybe at home, maybe in hospital, but as we're trying to get people more and more at home I would have thought that people want to be at home if at all possible really.'

Telephone interview 1: specialist nurse

'We're all for having a clinical nurse specialist to [deliver symptom management NPIs] but, actually, our CNS can't manage to get to clinics. So there's two issues: One is actually having the allied health professionals to be able to do these things, and the second thing is actually having space. There is no space for any additional people in the clinics.'

Telephone interview 3: oncology consultant

\section{Group or individually based teaching of non-pharmacological interventions}

The FGD discussion on group or individual teaching was linked to the discussion of the site for the intervention as it was deemed unlikely that groups would be taught within patient's homes. Nevertheless, regardless of the site, the participants generally agreed that delivery would ideally take place on an individual rather than a group basis. Working with patients individually was said to be likely to increase the motivation of patients to attend sessions, better facilitate the personalisation of the intervention to individual needs, and arguably have greater impact upon patients. One physiotherapist who regularly facilitated NPI learning noted that while 
groups might facilitate greater interaction between patients, allowing them to share experiences, it may nevertheless be more difficult for the facilitator to fully perceive the needs of the individuals that constituted the group and provide ongoing assessment of their progress.

'People behave differently in groups and they don't always give you a true reflection about how they're feeling.... One to one compared to groups has a lot of difference in terms of success... I also think it's a lot more difficult to assess in a group setting.'

Telephone interview 4: physiotherapist

\section{Involving caregivers within the teaching of non-pharmacological interventions}

The participants broadly agreed that patients with lung cancer often rely heavily on informal caregivers for many daily living activities. Because patients were said to often find it difficult to retain all of the information with which they are provided, the participants also agreed that it was almost always helpful to have informal carers or other family members or friends involved in the patient's care. The participants said that caregivers can often feel isolated and impotent to help patients, and involving them in a patient's learning of NPI approaches tailored to specific needs would provide caregivers with an important role, particularly as they are best placed to go through the techniques with the patient at home when patients are likely to need them most. It was also discussed that carers are not always able to accompany patients to clinics, and very often patients do not have carers because they live alone.

'And the carers as well have a big impact in that they probably feel a little bit isolated, they're in the chair at the side of [the patient] when they're having treatment, they're not actively involved. And by teaching them deep breathing exercises, etc., that they can pass on and encourage the patients to do, I think that will make a big difference without a doubt.'

(FGD 4) CNS

\section{Discussion}

HCPs seem to welcome the development of a NPI to manage the breathlessness-cough-fatigue symptom cluster and provide important insights into possible challenges and opportunities with such an intervention. It became clear from the analysis that the views of the participants concerning the necessary skills required to deliver NPIs, the factors required for the intervention to be effective and the complex symptomatology of the disease strongly suggested that the intervention should be personalised for each patient. This in turn had implications for other elements of the delivery method, and study participants broadly agreed upon several features. Firstly, it is most appropriate for the intervention to be delivered by a HCP rather than a trained volunteer or lay person, preferably a physiotherapist or specialist nurse. In order to personalise the NPI to patients according to their symptomatology, the participants found it essential that practitioners be aware of the underlying pathology of a patient's disease and the side effects of their medical treatment and be able to assess the effectiveness of the intervention for each individual. Evidence from previous studies emphasised the complexity of symptom management strategies and argued that those delivering such techniques should have some advanced knowledge and skills [46]. Within previous symptom management trials, the delivery of NPIs for patients with lung cancer has been the remit of both specialist nurses [4, 10,37] and physiotherapists [8, 22]. Nevertheless, it has also been argued that NPI teaching should not be regarded as the exclusive remit of any one particular group of HCPs but rather that all practitioners should incorporate it into their everyday practice [25]. Moreover, there should be recognition that translating research findings into existing clinical roles often encounters numerous difficulties for which staff require sufficient support $[25,41]$.

Secondly, the participants agreed that patients should receive the intervention when they were psychologically receptive, which required them to be symptomatic yet fit enough to practice the techniques before symptoms became severe. In UK, patients with cancer have been shown to present late and do badly [24], while the period between diagnosis and the point when symptoms become severe can often be short. This would suggest that only a small window of opportunity exists for patients to learn the various techniques. Nevertheless, despite these concerns expressed by the FGD participants, previous studies have been able to work successfully with some patients whose symptoms severely affected function, although other patients did drop out of the study $[4,10]$.

The third feature of a symptom management NPI that most HCP participants, whether community- or acute-based, agreed upon was that community staff would be better placed to deliver the intervention closer to patients' homes rather than having it be hospital-based. It could be difficult to motivate patients to make further hospital visits, especially with considerations of affordable transport and the deterioration of patients' health, and it has previously been suggested that staff in the community might be better placed to deliver NPIs [25]. Moreover, providing the intervention in the community rather than the acute sector is in line with the general direction of cancer care policy [14]. Fourthly, and in part related to this, most participants argued that the intervention be delivered to patients on an individual basis rather than in groups since a one-to-one intervention would better facilitate personalisation 
to individual patient needs and could motivate patients to attend. Finally, the participants agreed that, where possible, caregivers should be involved with the training and delivery of the intervention, not least because they were best placed to reinforce its use at home. Indeed previous research has shown that patients sometimes only attended symptom management clinics at the urging of family members [18].

Amongst the key issues that need to be addressed in the modelling of any complex intervention, prior to testing its effectiveness within a randomised controlled trial, is the identification of the underlying mechanisms by which the intervention could be best delivered and any potential constraints on its effectiveness in practice $[38,39]$. This modelling process is of particular importance for research in cancer care where patient attrition can be very high due to death or cognitive and physical deterioration $[1,27,30,44,46]$ and where recruitment relies heavily upon cooperation with HCPs who may be reluctant to provide researchers with patient access due to the high and complex symptomatology and low performance status often experienced by patients with cancer [6, 19, 44]. Such difficulties can lead to small sample sizes that limit the power of studies and the ability to generalise results: a problem affecting previous studies of NPI symptom management [46]. It has also been argued that the complex and intensive nature of NPIs may also raise issues about the feasibility of implementing them in routine clinical practice [46]. Thus, involving HCPs in the modelling phase of an intervention may help to reduce 'excessive gatekeeping' [44]. The approach taken within this study, therefore, was intended to minimise the potential problems with running any subsequent trial and the eventual implementation of the intervention in practice by incorporating the views of key stakeholders within its design. Although this paper reports the views of a necessarily broad range of HCPs, these views would need to be balanced with an objective assessment of previous research findings. Hence, this information combined with the patient and caregiver views can become the basis for developing a feasible, appropriate, sustainable and patient-centred intervention.

Acknowledgements The research team would like to especially thank those consultants and senior nurses who assisted with the recruitment of study participants: Dr. Carol Davies (SUHT), Sharon Woolley (Christie) and Dr. Pooja Jain (CCO). We would also like to thank our service user partners who have supported this research, John Belcher and Helen Walsh (who sadly both have died recently), and the National Cancer Research Institute for funding this study.

\section{References}

1. Ahlner-Elmqvist M, Bjordal K, Jordhoy MS, Kaasa S, Jannert M (2009) Characteristics and implications of attrition in health-related quality of life studies in palliative care. Palliat Med 23:432-440

2. Bailey CD, Wagland R, Dabbour R, Caress A, Smith J, Molassiotis A (2010) An integrative review of systematic reviews related to the management of breathlessness in respiratory illnesses. BMC Pulm Med 10:63

3. Bausewein C, Booth S, Gysels M, Higginson I (2008) Nonpharmacological interventions for breathlessness in advanced stages of malignant and non-malignant diseases. Cochrane Database Syst Rev 16(2):CD005623

4. Bredin M, Corner J, Krishnasamy M, Plant H, Bailey C, A'Hern R (1999) Multicentre randomised controlled trial of nursing intervention for breathlessness in patients with lung cancer. BMJ 318:888-889

5. Chan CW, Richardson A, Richardson J (2005) A study to assess the existence of the symptom cluster of breathlessness, fatigue and anxiety in patients with advanced lung cancer. Eur J Oncol Nurs 9:325-333

6. Cook AM, Finlay IG, Butler-Keating RJ (2002) Recruiting into palliative care trials: lessons learnt from a feasibility study. Palliat Med 16:163-165

7. Cooley ME (2000) Symptoms in adults with lung cancer: a systematic research review. J Pain Symptom Manage 19:137-153

8. Connors S, Graham S, Peel T (2007) An evaluation of a physiotherapy led non-pharmacological breathlessness programme for patients with intrathoracic malignancy. Pulm Med 21:285-287

9. Corner J, Plant H, Warner L (1995) Developing a nursing approach to managing dyspnoea in lung cancer. Eur J Cancer Care 8:30-36

10. Corner J, Plant H, A'Hern R, Bailey C (1996) Non-pharmacological intervention for breathlessness in lung cancer. Palliat Med 10:299 305

11. Cramp F, Daniel J (2008) Exercise for the management of cancerrelated fatigue in adults. Cochrane Database Syst Rev, Issue 2: CD006145

12. Degner LF, Sloan JA (1995) Symptom distress in newly diagnosed ambulatory cancer patients and as a predictor of survival in lung cancer. J Pain Symptom Manage 10:423-431

13. Department of Health (2000) The NHS cancer plan. Department of Health, London

14. Department of Health (2008) The cancer reform strategy. Department of Health, London

15. Dodd MJ, Miaskowski C, Lee KA (2004) Occurrence of symptom clusters. J Natl Cancer Inst Monogr 32:76-78

16. Dorman S, Jolley C, Abernethy A, Currow D, Johnson M, Farquhar M, Griffiths G, Peel T, Moosavi S, Byrne A, Wilcock A, Alloway L, Bausewein C, Higginson I, Booth S (2009) Researching breathlessness in palliative care: consensus statement of the National Cancer Breathlessness Subgroup. Palliat Med 23:213-227

17. Ellis J, Wagland R, Tishelman C, Lloyd Williams M, Bailey CD, Haines J, Caress A, Lorigan P, Smith J, Booton R, Blackhall F, Molassiotis A (2012) Considerations in developing and delivering a non-pharmacological intervention for symptom management in lung cancer: the views of patients and informal caregivers. J Pain Symptom Manage (in press)

18. Escalante CP, Kallen MA, Valdres RU, Norrow PK, Manzullo EF (2010) Outcomes of a cancer-related fatigue clinic in a comprehensive cancer centre. J Pain Symptom Manage 39:691-701

19. Ewing G, Rogers M, Barclay S, McCabe J, Martin A, Todd C (2004) Recruiting patients into a primary care based study of palliative care: why is it so difficult? Palliat Med 18:452-459

20. Fox SW, Lyons DE (2006) Symptom clusters and quality of life in survivors of lung cancer. Oncol Nurs Forum 33:931-936

21. Gift AG, Jablonski A, Stommel M, Given CW (2004) Symptom clusters in elderly patients with lung cancer. Oncol Nurs Forum 31:202-212

22. Hately J, Laurence V, Scott A, Baker R, Thomas P (2003) Breathlessness clinics within specialist palliative care settings can improve the quality of life and functional capacity of patients with lung cancer. Palliat Med 17:410-417

23. Henoch I, Pioner A, Tishelman C (2009) Increasing stringency in symptom cluster research: a methodological exploration of symptom 
clusters in patients with inoperable lung cancer. Oncol Nurs Forum 36(6):E282-E292

24. Holmberg L, Sandin F, Bray F et al (2010) National comparisons of lung cancer survival in England, Norway and Sweden 20012004: differences occur early in follow up. Thorax 65:436-441

25. Johnson M, Moore S (2003) Research into practice: the reality of implementing a non-pharmacological breathlessness intervention into clinical practice. Eur J Oncol Nurs 7:33-38

26. Joyce M, Schwartz S, Huhmann M (2008) Supportive care in lung cancer. Sem Oncol Nurs 24:57-67

27. Jordhøy MS, Kaasa S, Fayers P, Ovreness T, Underland G, AhlnerElmqvist M (1999) Challenges in palliative care research; recruitment, attrition and compliance: experience from a randomized controlled trial. Palliat Med 13:299-310

28. Kangas M, Bovbjerg DH, Montgomery GH (2008) Cancer-related fatigue: a systematic and meta-analytic review of non-pharmacological therapies for cancer patients. Psychol Bull 134:700-741

29. Krueger RA, Casey MA (2000) Focus groups: a practical guide for applied research, 3rd edn. Sage, Thousand Oaks

30. Ling J, Rees HJ (2000) What influences participation in clinical trials in palliative care in a cancer centre? Eur J Cancer 36:621626

31. MacDougall C, Fudge E (2001) Planning and recruiting the sample for focus groups and in-depth interviews. Qualitat Health Res $11: 117-126$

32. Miaskowski C, Dodd M, Lee K (2004) Symptom clusters: the new frontier in symptom management research. J Natl Cancer Inst Monogr 32:17-21

33. Molassiotis A, Wengström Y, Kearney N (2010) Symptom cluster patterns during the first year after diagnosis with cancer. J Pain Symptom Manage 39:847-858

34. Molassiotis A, Bailey C, Caress A, Brunton L, Smith J (2010) Interventions for cough in cancer. Cochrane Database Syst Rev. September 8: CD007881
35. Molassiotis A, Lowe M, Blackhall F, Lorigan P (2011) A qualitative exploration of a respiratory distress symptom cluster in lung cancer: cough, breathlessness and fatigue. Lung Cancer 71:94-102

36. Molassiotis A, Lowe M, Ellis J, Wagland R, Bailey C, Lloyd Williams M, Tishelman C, Smith J (2011) The experience of cough in patients diagnosed with lung cancer. Support Care Cancer 19:1997-2004

37. Moore S, Corner J, Haviland J, Wells M, Salmon E, Normand C et al (2002) Nurse led follow up and conventional medical follow up in management of patients with lung cancer: randomised trial. BMJ 325:1-7

38. MRC (2000) Framework for the development and evaluation of RCTs for complex interventions to improve health. Medical Research Council, London

39. MRC (2008) Developing and evaluating complex interventions: new guidance. Medical Research Council, London

40. NCCAC (2008) Diagnosis and treatment of lung cancer. National Collaborating Centre for Acute Care, London. Available from www.rcseng.ac.uk

41. Plant H, Bredin M, Corner J (2000) Working with resistance, tension and objectivity: conducting a randomised controlled trial of a nursing intervention for breathlessness. J Nurs Res 5:426-436

42. Rainbird K, Perkins J, Sanson-Fisher R, Rolfe I, Anseline (2009) The needs of patients with advanced, incurable cancer. Br J Cancer 101:759-764

43. Ritchie J, Lewis J (2003) Qualitative research practice: a guide for social science students and researchers. Sage, London

44. Ross C, Cornbleet M (2003) Attitudes of patients and staff to research in a specialist palliative care unit. Palliat Med 17:491-497

45. Shuy RW (2002) In-person vs telephone interviewing. In: Gubrium JF, Holstein JA (eds) Handbook of interview research: context \& method, chapter 26. Sage, London

46. Zhao I, Yates P (2008) Non-pharmacological interventions for breathlessness management in patients with lung cancer: a systematic review. Palliat Med 22:693-701 